\title{
OVERVIEW OF THE WORLD CONGRESS ON CORD BLOOD AND INNOVATIVE APPROACHES TO THE TREATMENT OF SICKLE-CELL ANEMIA IN MONACO ON 24-27 ${ }^{\mathrm{TH}}$ OCTOBER 2013
}

October 24-27 $7^{\text {th }}, 2013$ the IV World Congress on Cord Blood and Innovative Approaches to the Treatment of Sickle-Cell Anemia (ESH / Eurocord-Ed / Eurocord World Cord Blood Congress IV) was held at the Congress Center of Monaco under the patronage of Prince Albert II of Monaco.

Professor of Paris University Eliane Gluckman, who performed the world's first transplant of umbilical cord blood traditionally was the organizer of this event. This congress was special because dedicated to the $25^{\text {th }}$ anniversary of the first application of umbilical cord blood, which took place just in October 1988 in the hospital of St. Louis in Paris for the treatment of a child with Fanconi anemia.

The Congress was attended by about 300 participants from different countries from all continents, among whom were both world-renowned scientists - pioneers of cord blood application in medicine (E. Gluckman, D. Kurtzberg, H. Broxmeyer, D. Wagner, A. Madrigal), and medical practitioners and representatives from cord blood banking industry. The Congress was also attended by representatives of the Pontifical Academy of Life from Vatican, as the clinical use of cord blood is approved by all religions of the world as completely ethical source of adult stem cells.

Since 1988, more than 30000 transplants of hematopoietic stem cells of umbilical cord blood were performed worldwide, which have shown that cord blood is not only comparable with bone marrow as a hematopoietic stem cell transplant, but also has several important advantages, especially - a lower risk of «graft-versus-host» disease even in case of incomplete immunological matching. Umbilical cord blood is also regarded as an important source of mesenchymal stem cells that can be used both for immunosuppression in the treatment of the "graftversus-host» disease and a number of autoimmune disorders (multiple sclerosis, Crohn's disease, rheumatoid arthritis, systemic sclerosis, systemic lupus erythematosus) and as a biological material for tissue engineering. Among uptodate immunobiological preparations a special place belongs to the umbilical cord blood T cells. The scientists from all over the world make attempts to use them in specific immunotherapy of cancer and a number of viral and autoimmune diseases. The umbilical cord blood endothelial progenitor cells are also of huge interest for practical medicine, the use of these cells for the purposes of neovasculogenesis in vitro and in vivo is considered to be extremely promising. In terms of modern science, the umbilical cord blood is considered as an ideal source for iPS-cells for the discovery of which the scientists Shinya
Yamanaka and John Gurdon received the Nobel Prize in Medicine in 2012. More and more researchers pay attention to the value of cord blood plasma as a source of many growth factors and their potential regenerative and healing effect, as well as a substrate for the production of artificial tears.

In recent years, the umbilical cord tissue, as an attractive source of mesenchymal stem cells also attracts attention of the researchers that was repeatedly mentioned by the Congress speakers. In many countries of the world the protocols of isolation of mesenchymal stem cells from the umbilical cord tissue were developed and services of the long-term banking of these cells were brought to the market and a number of clinical studies on the effectiveness of these cells in the treatment of diseases were announced. A group of scientists from Greece under the supervision of C. Stavropoulos-Gioskas presented to Congress a new and interesting approach on the use of decellularized umbilical arteries as tissue scaffolds for tissue engineering.

However, despite significant advances in umbilical cord blood hematopoietic stem cell transplantation in the treatment of cancer and other diseases in children and adults, the following important problems remain in this field:

- the problem of a longer period of engraftment, compared to the adult bone marrow or peripheral blood stem cells;

- a small number of stem cells in a graft considering the treatment of a patient with a big body weight, if a cord blood sample of a small volume is collected;

- the frequent need for double cord blood transplants in the treatment of hematological malignancies in adults, which increases the cost of treatment.

Therefore, for the wider introduction of umbilical cord blood hematopoietic stem cell transplantation to clinical practice, especially in terms of insurance system of health care, scientists have to solve such matters as improving of cord blood engraftment and overcoming the problem of low-cell samples. But the scientists worldwide are actively developing protocols of cord blood stem cells expansion and genetic or pharmacological pretransplant manipulation of a graft aimed at improvement of the engraftment of hematopoietic stem cells.

A more detailed overview of the most important papers presented at the IV World Congress On Cord Blood And Innovative Approaches To The Treatment Of Sickle-Cell Anemia (ESH / Eurocord-Ed / Eurocord World Cord Blood Congress IV), is provided below. 
H. Broxmeyer (Indiana University, USA), in his report "Cord blood hematopoietic stem cells: State of the art including methods for graft facilitation" said that transplantation of hematopoietic stem cells of umbilical cord blood has become the procedure, saving lives of patients with malignant and nonmalignant diseases that require replacement of hematopoietic system. Improved protocols of freezing of cord blood samples and improved conditioning modes support a broad application of cord blood in hematological clinics. However cord blood transplantation still is accompanied by a sustained period of graft engraftment and immune recovery compared to adult bone marrow or hematopoietic peripheral blood stem cells. Scientists partially solved these problems, having developed a method of intrafemoral introduction of umbilical cord blood hematopoietic stem cells, as well as through expansion of cells ex-vivo, which is successfully carried out in many laboratories. The techniques of molecular manipulations of cells before transplantation to the recipient are also promising. Thus, according to the data of $\mathrm{H}$. Broxmeyer, actually the efficacy of such methods of stimulation of umbilical cord blood hematopoietic stem cells engraftment as the use of inhibitors of the enzyme dipeptidyl peptidase and use of moderate hyperthermia for modifying the expression of CXCR4 was shown.

G. Koegler (Institute of Transplantation Diagnostics and Cell Therapeutics, Dusseldorf, Germany) in the report «Cord blood subpopulations and reprogrammed cells" noted that cord blood stem cells are actively explored in preclinical studies aimed at the regeneration of cartilage tissue and treatment of cardiovascular diseases, including myocardial infarction, as well as diseases of the nervous system and liver failure . Professor drew attention to the prospects of the use of endothelial progenitor cells, mesenchymal stem cells, unrestricted somatic stem cells of umbilical cord blood, as well as fibroblast stem cells from umbilical cord tissue. The immunophenotypic characteristics of these cells are functionally different from the bone marrow cells. G. Koegler also noted that cord blood stem cells are genetically more immature than the cells from other adult sources and said, that the umbilical cord blood is considered to be the best source for iPS-cells. Potential use of the latter in regenerative therapy is considered to be very promising, but the professor emphasized the need for extensive research before introduction of $i P S$-cells to clinical practice. From the standpoint of G. Koegler, clinical use of iPS-cells is accompanied by the risk of structural failure of normal genes and activation of tumorigenesis. In 2013, in Japan the first clinical study on the safety and efficacy of $i P S$-cells in the treatment of agerelated macular degeneration launched.

Elizabeth Shpal (Texas University, USA) in a report entitled «Improved engraftment with cord blood expansion and fucosylation» said, that umbilical cord blood is an attractive source of hematopoietic stem cells for patients who need a transplant in the absence of an adult donor. Special value of umbilical cord blood is ethnic diversity of samples in global cord blood banks, simplicity of the collection, readiness for use and lower incidence and severity of the "graft-versus-host» disease as well as affordability of higher level of $H L A$ mismatching compared with the bone marrow or peripheral blood of an adult donor, although delayed engraftment. Professor presented the results of a clinical study on the efficacy of transplantation of two cord blood samples, one of which was expanded. Thus, according to the data of Elizabeth Shpal the average period of neutrophil and platelet engraftment in this case was 15 days (9-42) and 40 days (13-62), respectively. Transplantation of multiplicated cells was not accompanied by toxic effects. In 31 patients (97\%) and $26(81 \%)$ neutrophils and platelets were engrafted. The lecturer concluded that the expansion of stem cells is applicable and leads to faster engraftment of neutrophils and platelets and is characterized by a high level of independence from the infusion of platelets. It was reported that the problem of delayed engraftment of $C D 34^{+}$cells of cord blood can be solved by the use of recombinant fucosyltransferase enzyme. For example, the University of Texas initiated a clinical study on this therapeutic approach.
Isabelle Andre-Schmutz (Hopital Necker Enfants Malades, Paris, France) said that cord blood transplantation is an important therapeutic procedure for patients, who do not have an HLA-matched donor. Although umbilical cord blood as a source of hematopoietic stem cells has long been considered to be applicable only in pediatrics, the use of two samples allowed this method of treatment to acquire widespread in adult patients. One of the most important problems of hematological transplantation is the restoration of T-immunity and thymopoiesis of the recipient, as well as infectious complications in the post-transplant period. It was found that T-cells of donor origin appear in the thymus of recipients 9 months after transplantation of hematopoietic stem cells of umbilical cord blood, although complete restoration of immunity lasts longer and never returns to its original level. I. Andre-Schmutz considered a new therapeutic approach, which aims to stimulate the development of $T$ cells de novo generated by the introduction of in vitro T-cell progenitors. The latter are ready for the immediate subsidence in the thymus and the rapid generation of polyclonal T cells tolerant to the recipient organism.

Mary Eapen (Center for International Blood and Marrow Transplantation, Milwaukee, USA) presented an interesting paper on «Role of high resolution HLA typing for cord blood transplantation». Rapporteur overviewed 1,568 transplantations of umbilical cord blood hematopoietic stem cells for the treatment of malignant blood diseases, analyzing compatibility due to alleles $H L A-A,-B,-C$ and $D R B 1$. The results of this study confirmed the need for compatibility by HLA system when selecting a sample of umbilical cord blood for transplantation. Mary Eapen concluded that the incompatibility due to one or two loci of $H L A$ are tolerated better by the recipients than incompatibility due to 3,4 or 5 loci, which should be avoided in clinical practice.

Machteld Oudshoorn (Europedonor Foundation, Netherlands) presented a report on «Increasing use of hematopoietic stem cell transplantation for ethnic minorities" saying that the number of unrelated donors and cord blood transplants available in the world now makes over 22 million. This allows thousands of patients to receive unrelated hematopoietic stem cell transplant. However, considerable polymorphism of HLA system even in modern conditions makes impossible compatible unrelated donor selection for each patient. Most of voluntary donors of hematopoietic stem cells, registered in the world registers are of North-Western origin. So constant and unresolved problem of the organization of registers of hematopoietic stem cells is to attract donors from ethnic minorities, especially the people of India, China, Brazil, Nigeria. It is known that $93 \%$ of the available stem cell transplants are suitable for the representatives of Caucassian race. Cord blood banking in the recent decade has significantly increased the chances of finding a compatible transplant for patients from ethnic minorities. So, it is estimated that for the period from 2006 to $201045 \%$ of unrelated hematopoietic stem cells tranplantations for ethnic minorities were performed using umbilical cord blood.

Etienne Baudoux from the laboratory of genetics and cell therapy (Brussels, Belgium) in the report «Cord blood banking: State of the art» noted that since 1990 when the world's first cord blood bank was founded, the industry has grown significantly. So, now more than 590000 samples are stored in the public cord blood banks worldwide, that are able to replace 22 million of adult volunteer donors willing to donate their bone marrow for allogeneic transplants. Lecturer also considered the new tasks that face the industry of hematopoietic stem cells banking to date and among the primary ones named:

- stricter regulation of the industry in the U.S. and Europe;

- updating of professional standards;

- the presence of competing approaches to hematopoietic stem cell transplantation: easy access to adult unrelated donors, haploidentical transplants and the ability to carry out transplantation of hematopoietic stem cells from umbilical cord blood of several samples; 
- the safe collection of umbilical cord blood, taking into account the possible impact of this procedure on iron metabolism in newborns;

- determination of clear criteria for the selection of the graft $(H L A$, cell content);

- the role of cord blood banks for the production of drugs for use not only in hematology (iPS-cell immunotherapy, treatment of HIV);

- financial limitations.

G. Koegler (Dusseldorf, Germany) in another report entitled «Assessment of parameters influencing the quality of cord blood units» summarized that actually the quality of umbilical cord blood transplants is determined by the content of $C D 34$-positive hematopoietic progenitor cells, stem cell viability determined by flow cytometry and the ability of stem cell to proliferation. However, professor said, that these parameters do not allow to predict the ability of transplant engraftment. The report dealt with such methods of determination of the viability of stem cells as the use of annexin $V$ and the definition of colony-forming units. In laboratory practice, for determination of cell viability such methods as staining with 7-amino-actinomycin or propidium iodide are widely used, which enable to determine the number of dead cells. These stains react with DNA, but do not reveal the cells with damaged membrane and apoptotic cells. From the viewpoint of $G$. Koegler for the determination of extracellular expression of phosphatidylserine, caused by apoptosis, the use of Annexin $\mathrm{V}$ is preferred, while the reduction in the functionality of hematopoietic stem cells in association with apoptosis can be identified by the ability of cells to form colonies. Results of assays using annexin $\mathrm{V}$ revealed that even in fresh samples of umbilical cord blood (shelf life at least 36 hours), more than $10 \%$ of the cells were apoptotic due to the procession effect. However, staining with 7-aminoactinomycin or propidium iodide did not show it. Attention was also drawn that the effect of the cryoprotectant dimethyl sulfoxide increases apoptosis of stem cells. Thus, the number of cells in a sample, suitable for transplantation, is actually lower than it is possible to determine by conventional methods.

Sergio Querol, director of the cord blood bank of Barcelona (Barcelona, Spain) in the report «Updating the cord blood unit inventory to meet current needs» said, that the use of unrelated cord blood transplants widely began in 1993, initially only in pediatrics and then in adults patients. The lecturer cited statistics according to which the number of cord blood transplants performed in children has remained stable since 2007 and is about 1,250 transplants per year. At the same time, according to the World Marrow Donor Association (WMDA), the number of transplants of hematopoietic stem cells of umbilical cord blood in adult patients in the world has actually grown to 2,750 transplants per year. In 2012, there was a slight decrease in the number of cord blood transplants performed in Europe and the U.S., but an increase of transplant activity with the use of umbilical cord blood in Japan (1000 transplants/year), Korea, France, Mexico and the United Kingdom of Great Britain and Northern Ireland was noted. Currently the growth of cord blood banking industry is about $10 \%$ per year. S. Querol also considered demands to modern umbilical cord blood banks and the following criteria, which they must comply:

\section{Safety:}

- justification of the suitability of the sample for clinical use, the risks, the history of transportation;

- laboratory tests (serum and NAT HBV, HCV, HIV, HTLV, CMV $E B V$, Toxoplasma analysis, bacteria, fungi, genetic screening of hemoglobinopathies.

2. Identity:

- $\quad C B$ HLA-A,-B,-C,-DRB1. Maternal HLA-A,-B, DRB1 low resolution;

- $A B O$ and gender;

- Analysis of reference samples: maternal/cord plasma and serum, DNA samples;
- Analysis of the sample from a satellite (at least one, which is transported together with the basic pattern).

3. Purity:

- the dose and the number of CD34 cells and red blood cells after treatment.

\section{Potency:}

- clonogenic ability of CD34-cells $>10 \%$;

- viability (7AAD) more $85 \%$

- viability of a satellite sample after thawing.

The lecturer, as the previous speakers, also justified the necessity of cord blood banking for ethnic minorities.

V. Rocha (University of Oxford, Oxford, UK), scientific director of the international organization EUROCORD, established at the European Group for Blood and Marrow Transplantation (EBMT) to regulate cord blood banking and transplantation, as well as statistical monitoring of the industry, in his reports considered current activity of EUROCORD. To date, the organization includes two divisions, actually register of EUROCORD, collaborating with 16 banks of umbilical cord blood, which is subordinate to the Ministry of Health of France, and the research team, which is based in St. Louis Hospital in Paris, where in 1988 the world's first cord blood transplant has been performed. Professor emphasized that the success of the clinical application of umbilical cord blood is closely linked with the industry of banking of this biological material. The most important criteria regarding the selection of the sample of umbilical cord blood for transplantation to date are: HLA-phenotype, the total number of nucleated and $C D 34^{+}$cells as well as screening for infectious agents. Currently, more than 130 public cord blood banks are being operated in the world, which, as we mentioned above, store about 600000 cord blood sample, and as of 2012, 30000 unrelated umbilical cord blood transplantations were performed.

Within the work of the congress separate meetings were devoted to current issues of diagnosis and treatment of sickle cell anemia and the role of hematopoietic stem cell transplantation of umbilical cord blood in the therapy of this disease. So, the patriarch of cord blood application in medical practice

Eliane Gluckman (University of Paris, France) presented a paper «Sickle-cell disease - a global perspective». Sickle-cell anemia is an acute hereditary disorder, at which the abnormality in hemoglobin structure occurs. The absence of adequate treatment for sickle cell anemia leads to multiple organ failure and death in early childhood. Sickle cell anemia occurs mainly in countries, where malaria is prevalent - Africa, India, Brazil, Caribbean Islands, although it was noted that due to the migration of the modern population, currently sickle cell anemia has become increasingly common in other countries. According to statistics, every year about 350000 children with hemoglobinopathies are born worldwide, $80 \%$ of them in developing countries. Approximately $70000-100000$ persons with sickle cell disease live in the United States. Currently, each year about 400 cases of sickle-cell anemia are diagnosed in infants in France. In the 70 's years of the 20th century, $10 \%$ of Americans suffering from sickle-cell anemia died before 10 years of age, and $50 \%$ of patients did not survive till the age of 21. Today, the average life expectancy of a patient with sicklecell anemia is $40-50$ years. Significant progress in the treatment of sicklecell anemia has been achieved through the development of methods of pneumococci infection prevention, introduction of hydroxycarbamide into clinical practice and early detection of vasculopathies of cerebral vessels. However, the only way to cure this disease is HLA-identical transplantation of hematopoietic stem cells from a sibling. However, despite the high level of curing of sickle-cell anemia due to hematopoietic stem cell transplantation $(>90 \%)$, this treatment is not available to the majority of patients because of the low standard of living in their country or the lack of a donor. Therefore, Prof. Gluckman and other speakers stressed the importance of banking of cord blood transplants for patients with sickle cell disease, especially those belonging to ethnic minorities. 
J. Kurtzberg (Duke University, USA), who performed the world's first unrelated cord blood transplantation, presented an interesting report «Nicord expanded hematopoietic progenitor cells enhance hematopoiesis and facilitate cord blood engraftment after mieloablyative chemotherapy for patients with sickle-cell disease». Professor told about a new cell preparation Nicord, which is based on expanded hematopoietic stem cells from umbilical cord blood. It is shown that Nicord provides fast and efficient engraftment of hematopoietic stem cells of umbilical cord blood in adult patients in case of transplantation of two samples of umbilical cord blood in the treatment of high-risk hematological malignancies. Nicord also is regarded as a promising drug for the treatment of sicklecell anemia, which allows to solve the problem of lack of donors. Thus, a new therapeutic strategy for the treatment of sickle-cell anemia, which implicates the transplantation of nonmanipulated hematopoietic stem cells and umbilical cord blood drug Nicord, was considered in the report The United States have initiated a clinical study, which recruites patients with high risk sickle cell disease in the age from 2 to 21 years, for whom 2 samples of umbilical cord blood, suitable for transplantation are found. Hematopoietic stem cells from a first sample are administered to the patient without any manipulations, while the second sample undergoes special treatment for the production of drug Nicord. The graft consists of Nicord CD133-positive fraction of hematopoietic progenitor cells propagated for 21 days and uncultivated fractions of frozen and thawed CD133-negative T cells. Currently, according to this method 4 patients aged 8-13 years were treated, 1 of whom died after $2^{\text {nd }}$ transplantation because of the graft failure and 3 patients are alive for 2-13 months after transplantation.

J. Wagner of the University of Minnesota (USA) presented the report «Improving outcomes in recipients of unrelated umbilical cord blood State of the art» The scientist, as well as many other speakers noted that delayed immune reconstitution is an important issue in the transplantation of hematopoietic stem cells of umbilical cord blood. But the results of laboratory and clinical research on stem cell expansion of umbilical cord blood are promising. Professor considered the prospects of application of regulatory $T$ cells of umbilical cord blood for the prevention and treatment of the syndrome of "graft-versus-host" in oncology, which eliminates the need for post-transplant immunosuppression. It was found that propagated ex-vivo regulatory $T$ cells of umbilical cord blood have a high immunosuppressive effect, and their use is considered to be safe and can be implemented into clinical practice. The speaker also noted that pretransplantation conditioning causes damage to epithelial cells of the thymus of patients and negatively affects the recovery of T-lymphocyte immunity. D. Wagner introduced innovative therapeutic approach involving the infusion of progenitor thymocytes that allows to restore quickly the number and function of $T$ lymphocytes in recipients of hematopoietic stem cells.

A. Ruggeri (EUROCORD, Paris, France) in his report overviewed the effectiveness of transplantation of one or two samples of umbilical cord blood in adults with hematological malignancies. Professor said that the transplantation of two cord blood samples was first described in 2001 and this method has been implemented in clinical practice in several countries. Thanks to the introduction of a method of transplantation of two cord blood samples into clinical practice, the number of cord blood transplantations in adults today exceeded this index in children. So, there is evidence of 10000 transplants of hematopoietic stem cells from umbilical cord blood performed in adult patients. It is also important that the period of engraftment in case of transplantation of 2 cord blood samples, according to CIBMTR, is comparable with that using a single sample. EUROCORD also reported that transplantation of two cord blood samples is more effective compared with transplantation of one sample, although it is more expensive. However, according to experts, these costs are justified. At the same time, some scientists have voiced concerns about the higher risk of developing the syndrome of "graft-versus- host» in the case of transplantation of two cord blood samples.
A team of researchers from New York Medical College under the leadership of Dr. M. S. Kairo presented the results of experimental studies on the effectiveness of unrestricted somatic stem cells of cord blood for the treatment of dystrophic epidermolysis bullosa, demonstrating their high efficiency. Experimental studies in newborn mice showed that stem cells injected intrahepatically get quickly into the bloodstream, were not found in the internal organs (heart, kidneys, spleen, etc.), but were detected in lesions on the feet and in the gastrointestinal tract. This suggests specific migration of injected stem cells in place of wound defects. Interestingly, the intrahepatic infusion of stem cells were characterized by better results compared with infusions or intradermal administration of cells in the facial vein. The results showed that cell therapy of epidermolysis bullosa in the experiment improved survival in animals and promote wound healing.

P. Rebulla from the Foundation Ca 'Granda Ospedale Maggiore Poiliclinico (Milan, Italy) presented an interesting report on clinical investigations of wound healing gel, created on the basis of cord blood platelets, which is patented by Italian scientists. The drug has shown efficacy in the treatment of venous ulcers of lower limbs in diabetic patients. Compared with the same drug that is made from peripheral blood platelets from adult donors, platelet gel based on umbilical cord blood has several advantages. They include accessibility, lack of viral infections and a higher content of vascular endothelial growth factor. However, some obstacle to the commercialization of this drug is its high cost, as a course of treatment of one patient requires 50 cord blood samples.

M. S. Cairo (USA) also introduced a pilot project of study on the efficacy of unrelated cord blood transplantation and placental stem cells in children with malignant and benign diseases. It is now proven that conditioning and subsequent transplantation of hematopoietic stem cells of umbilical cord blood are safe and well tolerated. However, the relatively small number of hematopoietic stem cells in cord blood sample has prompted scientists to look at the placenta as another rich source of stem cells. Celgene Cellular Therapeutics developed a perfusion system for sterile obtaining of stem cells from the placenta of full-term pregnancies. Currently a clinical study was initiated to evaluate the safety and suitability of the placental stem cell transplantation in conjunction with transplantation of hematopoietic stem cells from one or more cord blood samples. Preliminary results show that this approach is safe and well tolerated.

Thus, 25 year experience of the clinical application of umbilical cord blood in hematology and results of high-tech studies on innovative approaches to the production of qualitatively new biological products based on cord blood once again confirm that this biological material took a firm place in the health care system in most countries of the world and has passed the test of the time. Widespread use of hematopoietic stem cells of umbilical cord blood in conditions of insurance health care system confirms the feasibility and economic justifiability of its long-term banking.

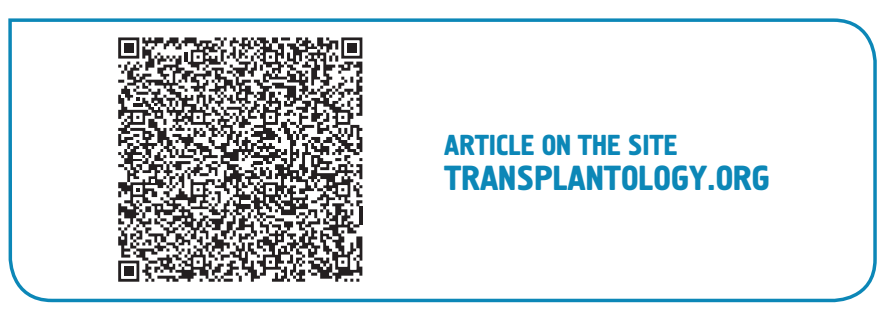

